\title{
HIGH-RESOLUTION MAGNETIC RESONANCE IMAGING OF THE NORMAL EXTRAOCULAR MUSCULATURE
}

\author{
A. ETTL ${ }^{1,2}$ J. KRAMER, ${ }^{3}$ A. DAXER ${ }^{4}$ and L. KOORNNEEF ${ }^{2}$ \\ St Pötten, Linz and Innsbruck, Austria and Amsterdam, The Netherlands
}

\begin{abstract}
SUMMARY
Purpose: Magnetic resonance imaging (MRI) of the extraocular muscles has attracted growing interest for the evaluation of complex motility disorders. However, little information is available on the high-resolution MRI anatomy of the normal extraocular muscles and their connective tissue system. The study describes the imaging anatomy of the recti and oblique muscles and the levator palpebrae superioris muscle.

Methods: MRI of the orbit at 1 tesla was performed in four normal volunteers using a surface coil.

Results: Many anatomical details such as Zinn's tendinous annulus, the trochlea, the superior oblique tendon, the intermuscular septa, the check ligaments, Lockwood's ligament and the common sheath between the superior rectus muscle and the levator muscle were visualised. A striking imaging feature was the curved path of both the recti muscles and the levator palpebrae muscle. The inferior oblique muscle also showed a marked curvature in the region of Lockwood's ligament.

Conclusions: High-resolution MRI is capable of demonstrating the anatomy of the extraocular musculature and parts of its connective tissue system. The curved path of the extraocular muscles can be explained by the configuration of the orbital connective tissue system which couples each extraocular muscle with the adjacent orbital wall. We discuss the clinical implications of our findings and review previous radiological studies regarding the functional anatomy of the extraocular muscles.
\end{abstract}

From: ${ }^{1}$ Department of Orthoptics, General Hospital, St Pölten, Austria; ${ }^{2}$ Orbital Center, Academic Medical Center, University of Amsterdam, The Netherlands; ${ }^{3} \mathrm{CT} \&$ MRI Institute, Linz, Austria; ${ }^{4}$ Department of Ophthalmology, University of Innsbruck, Austria.

Correspondence to: Dr. Armin Ettl, Abteilung für Orthoptik, Allgemeines Krankenhaus, Postfach 176, A-3100 St Pölten, Austria. Tel: +43-2742-300-2869 (hospital), +43-2742-340802 (private office). Fax: $+43-2742-3285$.
Magnetic resonance imaging (MRI) is a good method for evaluating extraocular muscle disorders. ${ }^{1}$ Most publications focus on MRI of orbital disease, ${ }^{1}$ but do not provide enough information on normal orbital imaging anatomy. Since the early anatomical MRI studies of the orbit, ${ }^{2}$ advanced MRI technology has led to a marked improvement in the resolution of orbital MR images, although motion artifacts still represent a significant problem. The present study was performed to describe the MRI anatomy of the normal extraocular muscles under resting conditions in vivo.

\section{MATERIALS AND METHODS}

Six orbits from four normal volunteers aged from 26 to 32 years were examined. MRI was performed using a 1 tesla scanner (Impact, Siemens, Germany) and a surface coil with a diameter of $10 \mathrm{~cm}$. T1-weighted images of the orbit were obtained by spin-echo sequences with an echo time (TE) of $15 \mathrm{~ms}$ and a repetition time (TR) of 440-520 ms. Imaging planes included axial, coronal and sagittal (parallel to the optic nerve) sections. The slice thickness was 2-3 $\mathrm{mm}$ without any interslice interval. The field of view in the original images ranged between $140 \times 140 \mathrm{~mm}$ with a $256 \times 256$ matrix and $230 \times 230 \mathrm{~mm}$ with a $512 \times 512$ matrix. The acquisition time was 2-13 min. Images were taken with both lids closed and the eyes in resting position (slight downgaze). The structures in the MR images were identified by comparison with the collection of histological sections of the orbit by Koornneef. ${ }^{3}$

\section{RESULTS}

The recti muscles have their origin at Zinn's tendinous annulus in the orbital apex (Fig. 1). In the region between the equator and the posterior pole of the globe, the vertical and horizontal recti muscles are bowed away from the eye thus showing a curved path in the orbit (Figs. 1, 2). On coronal 


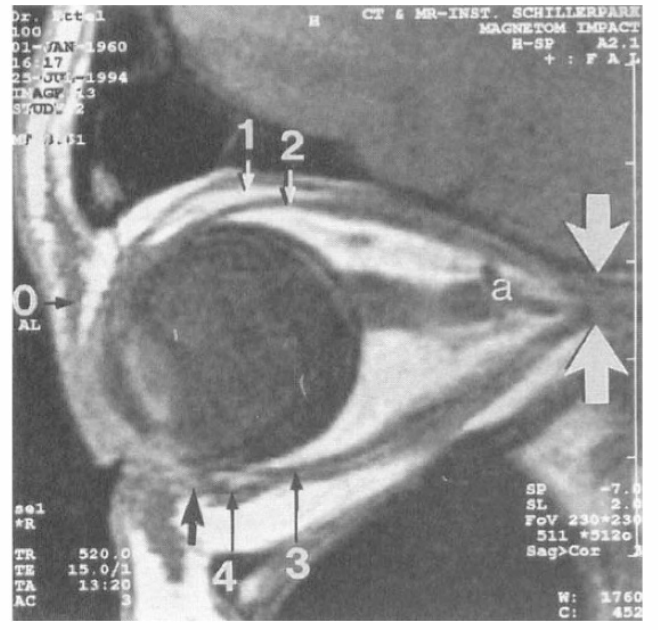

Fig. 1. Sagittal MR image (right orbit, resting position in slight downgaze - section through the optic nerve) showing the curved path of the levator palpebrae superioris (LPS) muscle (1), superior rectus (SR) muscle (2) and inferior rectus (IR) muscle (3). An intermuscular space containing the common fascia ('common sheath') of the SR and LPS muscles and adipose tissue is visible between the anterior LPS and the SR. The culmination point of the LPS is located a few millimetres behind and above the equator of the globe. Zinn's tendinous annulus (white arrows), the origin of the recti muscles, is seen at the orbital apex. The inferior oblique (IO) muscle (4) is seen $2 \mathrm{~mm}$ below the tendon of the IR. O, orbicularis muscle; black arrow, capsulopalpebral fascia; a, ophthalmic artery. (Modified with permission from [14].)

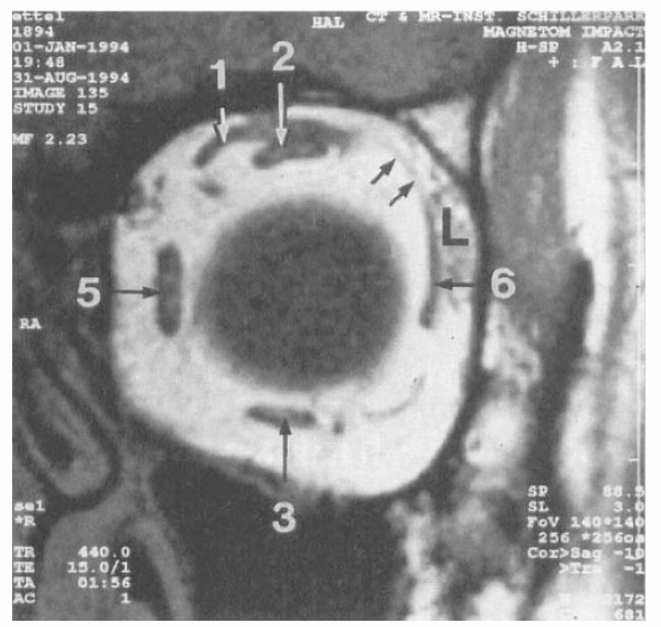

Fig. 3. Coronal $M R$ image (right orbit - section plane behind the equator) showing the recti muscles coursing at a distance from the globe. The superolateral intermuscular septum (arrows) connects the LR (6) with the superior muscle complex consisting of $S R$ (2) and LPS (1). 5, MR; 3, IR.

images just posterior to the equator, a gap between the globe and the recti muscles is noted which is due to the curved path of the muscles (Fig. 3). The line of tangency where the straight muscles start to touch the surface of the globe is located in the equatorial region or $2-3 \mathrm{~mm}$ behind the equator (Fig. 2). After the line of tangency, the straight muscles run in close

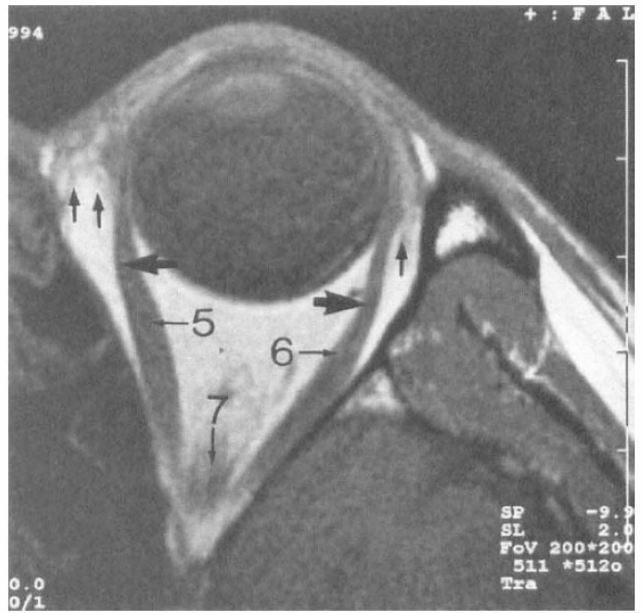

Fig. 2. Axial MR image (right orbit, resting position) demonstrating the curved path (thick arrows) of the medial rectus (MR) muscle (5) and lateral rectus (LR) muscle (6). The medial and lateral check ligaments (small arrows) connect the horizontal recti with the orbital walls. 7, IR.

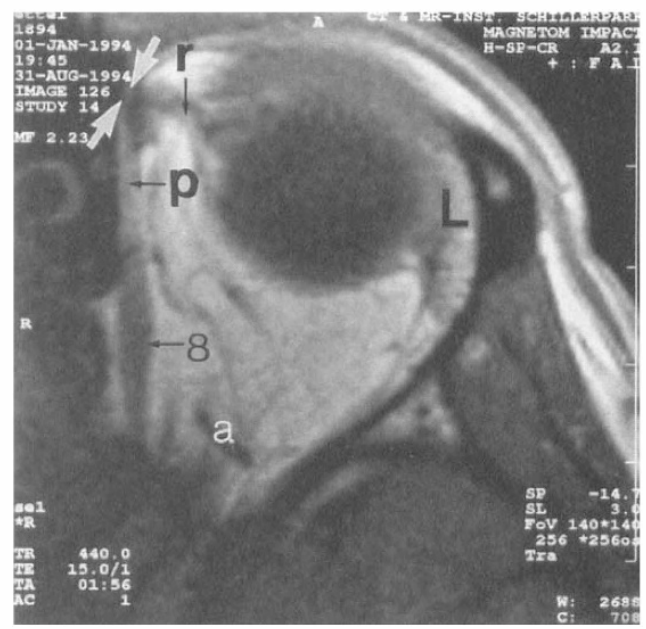

Fig. 4. Axial MR image (right orbit) at the level of the trochlea (white arrows) showing the belly of the superior oblique (SO) muscle (8), the reflected part of the SO tendon $(r)$ and the pretrochlear part of the SO tendon $(p) . L$, lacrimal gland; $a$, ophthalmic artery.

contact with the globe in a wide circular path ('arc of contact') towards their insertion. This segment of the muscles or their tendons respectively, cannot be clearly differentiated from scleral tissue on MRI.

The medial check ligament that attaches the medial rectus muscle (MR) to the medial orbital wall, is visualised on axial images (Fig. 2). Due to the 


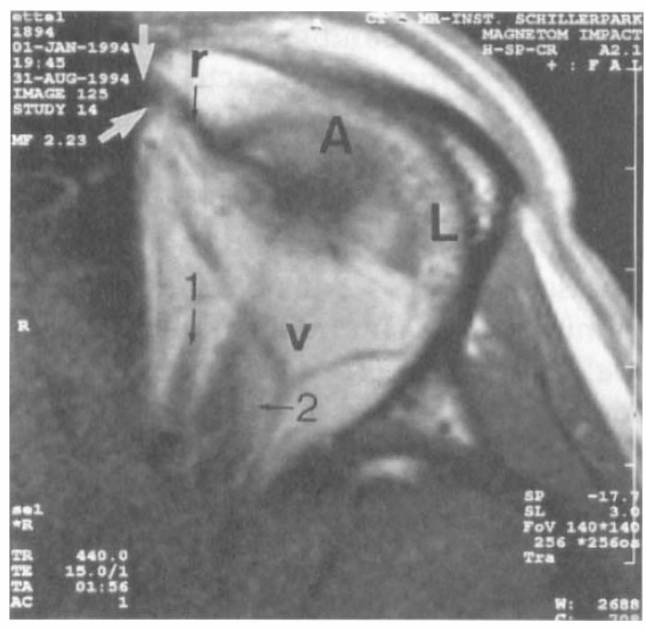

Fig. 5. Axial image (right orbit) showing the reflected part of the superior oblique tendon ( $r$ ) and the trochlea (white arrows). 1, LPS; 2, SR; A, levator aponeurosis; L, lacrimal gland; $V$, superior ophthalmic vein.

isointensity with lacrimal gland tissue, the lateral check ligament cannot be seen in mid-axial images. Low axial images show parts of the lateral check ligament (Fig. 2). Coronal sections behind the equator show the thick superolateral intermuscular septum. Weaker intermuscular septa connect the inferior rectus muscle with the medial and lateral recti muscles (Fig. 3). Septa coursing from the medial, inferior and lateral rectus muscles towards the orbital walls are also noted (hardly visible in the photographic reproductions).

The superior oblique muscle (SO) originates from the lesser wing of the sphenoid and courses in close contact with the superomedial orbital wall to the trochlea (Fig. 4). From there, the reflected part of the SO tendon courses postero-laterally in an estimated angle of about $45-55^{\circ}$ with the sagittal plane (Fig. 5) to insert in the superolateral quadrant of the globe. The trochlea is visualised in axial and coronal images (Figs. 4-6).

The inferior oblique muscle (IO) originates from the maxilla just lateral to the entrance of the nasolacrimal canal and runs posterolaterally to insert in the inferolateral quadrant of the globe under the inferior border of the lateral rectus muscle (Fig. 6). The IO belly appears in a cross-section approximately $2 \mathrm{~mm}$ below the inferior rectus (IR) on midsagittal images (Fig. 1). The lower lid retractors (capsulo-palpebral fascia and inferior tarsal muscle) originate from the anterior border of the IO and insert in the tarsal plate of the lower lid (Fig. 1).

On sagittal images, the levator palpebrae superioris muscle (LPS) courses upwards from its origin at the lesser wing of the spenoid until it reaches a culmination point about $3-5 \mathrm{~mm}$ (craniocaudal distance) superior to the equator of the globe, from where it courses downwards to the insertion in the

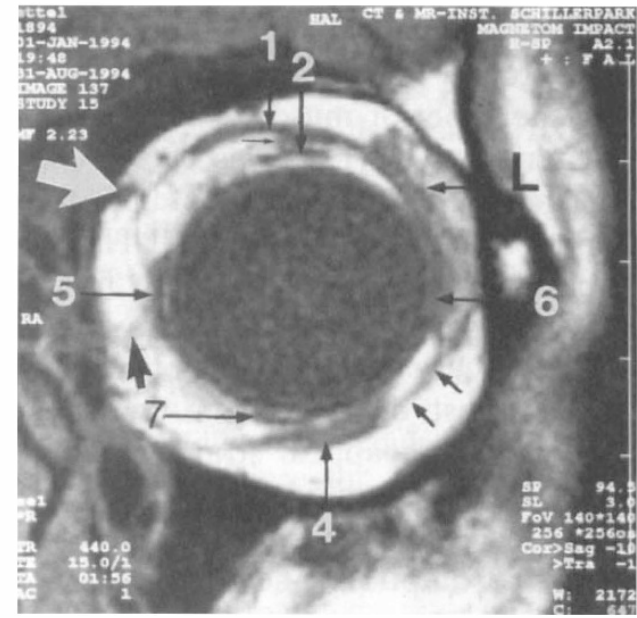

Fig. 6. Coronal MR image (right orbit - section approximately at the equator) showing the superior (2), inferior (7), medial (5) and lateral (6) rectus muscles, the levator muscle (1) and the trochlea (white arrow) in a cross-section. The inferior oblique muscle (4) is bowed away from the eye in the region of Lockwood's ligament. The medial check ligament (thick black arrow) connects the MR to the orbital walls. The inferolateral intermuscular membrane (pair of black arrows) is noted between the inferior muscle complex and the LR. The common sheath (thin arrow) is visible between the LPS and the SR. (Modified with permission from [14].)

upper lid. In the posterior and mid-orbit the LP is situated in close proximity to the orbital roof. In primary gaze or slight downgaze, the location of the culmination point of the LPS is located about 4-5 $\mathrm{mm}$ (anteroposterior distance) posterior to the equator of the globe. Between the culmination point of the LPS and the superior rectus (SR), a space that is isointense to orbital fat is noted. This intermuscular space also contains hypointense structures which are interpreted as strands of the common fascia of the LPS and the SR ('common sheath') (Fig. 1).

\section{DISCUSSION}

The origin and course of the extraocular muscles (EOM) can be demonstrated on MR images with sufficient detail. However, due to the varying arc of contact (region of tangency between the EOM and globe) and the isointensity of tendon and scleral tissue, an exact determination of the insertion of the recti and oblique muscles is not possible.

Koornneef was the first to describe the highly complex connective tissue system of the orbit. ${ }^{3-5}$ Due to the good contrast between hyperintense orbital fat and hypointense connective tissue structures, various parts of the connective tissue of the EOM were visualised on MRI: the so-called medial and lateral check ligaments, the common sheath between the SR muscle and levator muscle, Lockwood's ligament and its arcuate expansion, radial septa coupling the EOM with the orbital walls and intermuscular septa in the 
anterior orbit. In particular the superolateral intermuscular septum ('tensor intermuscularis muscle') connecting the superior muscle complex (LPS, SR) with the lateral rectus (LR) was clearly visible on coronal images due to its thickness of up to $1 \mathrm{~mm}$ and the content of striated muscle fibres. ${ }^{5}$ The thickness of the superolateral septum has been found to be enlarged in patients suffering from Graves' disease. ${ }^{6}$

In the past, it was believed that the recti EOM follow the shortest distance from their origin to insertion because they appear straight in anatomical specimens. Previous models of eye muscle mechanics, such as the 'Fadenmodel', ${ }^{7}$ have been based on this assumption. The MR images confirm the CT-based observation of Simonsz and co-work$\mathrm{ers}^{8}$ that the recti EOM do not follow the shortest path from their origin to the line of tangency but are bowed away from the retro-equatorial region of the eye. Simonsz's CT studies demonstrated that there is no significant sideways displacement (relative to the bony orbit) of the horizontal recti muscles during vertical eye movements or of the vertical recti muscles during horizontal eye movements. ${ }^{9}$ The course of the bellies of the recti EOM is hardly changed by surgical muscle transpositions. ${ }^{10}$ All these findings confirm that the path of the recti EOM is stabilised by means of the orbital connective tissue system..$^{3-5}$

The recti muscles enter sub-Tenon's space by passing through the fascial sleeves of Tenon's capsule. These fascial sleeves are attached to the medial and lateral orbital walls by means of connective tissue septa ('check ligaments') that contain smooth muscle cells. ${ }^{4}$ The smooth muscle cells in the check ligaments may serve to adjust the tension of the EOM. ${ }^{4}$ Demer and co-workers ${ }^{11}$ have recently suggested that the sleeves in Tenon's capsule together with the check ligaments represent fibromuscular pulleys for the recti EOM. The symmetrical arrangement of the rectus muscle pulleys is thought to be the mechanical basis of Listing's law. ${ }^{11}$ The anterior part of the SR is coupled to the superior periorbit via the common muscle sheath, which has connections to the orbital walls via the superior transverse ligament and the radial septal system.

Outside Tenon's capsule and behind the equator of the globe, the recti muscles and the SO belly are coupled to the orbital walls with connective tissue septa anchoring their fascial sheath to the periorbit. $^{3-5}$ The supporting framework of connective tissue septa around the EOM would explain their stability against sideways displacement during ocular movements and following surgical transpositions. ${ }^{10}$ The fibromuscular pulleys ${ }^{11}$ can be regarded as the functional origin of the EOM. The path of the eye muscles and the position of their functional origin is considered to be dependent on the following factors:
1. The muscle tension and gaze position. In primary or resting position, the recti muscles appear curved. However, straightening of a contracting muscle occurs if the eye is moved into the field of action of that muscle, as can be seen in the figures of a paper by Bailey et al. ${ }^{12}$

2. Intermuscular forces due to intermuscular septa that limit the side-slip ${ }^{9}$ of the muscles.

3. Musculo-orbital forces due to connective tissue suspensions of the muscles. ${ }^{11}$

4. Retrobulbar forces caused by the counter-pressure that is built up during muscle contractions.8

The contrast between the straight appearance of the recti EOM in dissection specimens and their curved path in imaging studies may be explained by the fact that during anatomical dissections where parts of the orbital walls have to be removed, the delicate connective tissue system of the muscles is destroyed.

The course of the oblique eye muscles is also determined by connective tissue structures. The trochlea, as the pulley of the SO, translates the antero-posterior muscle force of the $\mathrm{SO}$ into a downwards movement of the globe. After surgical disinsertion of the trochlea or luxation of the SO tendon out of the trochlea for treatment of Brown's syndrome, ${ }^{13}$ the SO belly does not significantly displace laterally but still maintains its proximity to the superomedial orbital wall as demonstrated by CT scans with three-dimensional reconstructions. Again, this stability against displacement can be explained by connective tissue septa anchoring the SO belly to the superomedial periorbit. ${ }^{3-5}$ The IO also appears to be pulled away from the globe in the region of Lockwood's ligament, which may therefore represent the 'pulley' of the IO. The pulley of the IR is located further posteriorly, as can be observed in sagittal images (Fig. 1). The IO is surrounded by a strong connective tissue complex, which explains why following a surgical disinsertion of the IO, the muscle rarely retracts further than to the IR.

The LPS muscle also follows a curved path in the orbit: it ascends towards a culmination point which is situated under the anterior orbital roof from where it descends towards the insertion in the upper lid. The location of the culmination point of the LPS a few millimetres superior to the globe suggests a suspension of the LPS by radial connective tissue septa coursing from the muscle to the orbital roof. The common sheath in addition to the globe may support the LPS from below, thus acting as a fulcrum for the LPS. ${ }^{14}$

Our study has described the MRI anatomy of the normal EOM. A thorough understanding of the normal morphology of the EOM is a prerequisite for the interpretation of MR images in orbital disorders. Recently, MRI has been applied to the evaluation of ocular motility disorders: chronically paretic muscles 
have a decreased cross-sectional area and are lacking normal contractile changes during different gaze positions. ${ }^{15}$ For example, this enables a differentiation between superior oblique palsy and hypertropia of other causes. Cine MRI, which involves MRI in different gaze positions to produce a video recording of ocular movements, ${ }^{12}$ has been used to analyse restrictive motility disorders. ${ }^{16}$

In our opinion, high-resolution MRI will soon find a place in clinical practice for the evaluation of complicated motility disorders in selected patients.

Key words: Magnetic resonance imaging, Extraocular muscles, Anatomy.

\section{REFERENCES}

1. DePotter P, Shields JA, Shields C. MRI of the eye and orbit. Philadelphia: Lippincott, 1995.

2. Langer B, Mafee MF, Pollack S, Spigos DG, Gyi Bo. MRI of the normal orbit and optic pathway. Radiol Clin North Am 1987;25:429-46.

3. Koornneef L. Spatial aspects of musculo-fibrous tissue in man. Amsterdam: Swets \& Zeitlinger, 1976.

4. Koornneef L. New insights in the human orbital connective tissue. Arch Ophthalmol 1977;95:1269-73.

5. Koornneef L. Orbital septa: anatomy and function. Ophthalmology 1979;86:876-9.

6. Goodall KL, Jackson A, Leatherbarrow B, Whitehouse RW. Enlargement of the tensor intermuscularis muscle in Graves' ophthalmopathy. Arch Ophthalmol 1995;113:1286-9.
7. Günther S. Modellmässige Beschreibung der Augenmuskelwirkung. Hamburg: University of Hamburg, Diplomarbeit, 1986.

8. Simonsz HJ, Haerting F, de Waal BJ, Verbeeten B. Sideways displacement and curved path of the recti eye muscles. Arch Ophthalmol 1985;103:124-8.

9. Miller JM, Robins D. Extraocular muscle sideslip and orbital geometry in monkeys. Vision Res 1987;27: 381-92.

10. Miller JM, Demer JL, Rosenbaum AL. Effect of transposition surgery on rectus muscle paths. Ophthalmology 1993;100:475-87.

11. Demer JL, Miller JM, Poukens V, Vinters HV, Glasgow BJ. Evidence for fibromuscular pulleys of the recti extraocular muscles. Invest Ophthalmol Vis Sci 1995;36:1125-36.

12. Bailey CC, Kabala J, Laitt R, Ho HB, Potts MJ, Harrad RA, Waston M, Goddard P. Cine magnetic resonance imaging of eye movements. Eye 1993;7: 691-3.

13. Mombaerts I, Koornneef L, Everhard-Halm Y, Hughes D, de Buy Wenninger-Prick L. Superior oblique luxation and trochlear luxation: new concepts in superior oblique muscle weakening surgery. Am J Ophthalmol 1995;120:83-91.

14. Ettl A, Priglinger S, Kramer J, Koornneef L. Functional anatomy of the levator palpebrae superioris muscle and its connective tissue system. Br J Ophthalmol 1996;80:702-7.

15. Demer JL, Miller JM. Magnetic resonance imaging of the functional anatomy of the superior oblique muscle. Invest Ophthalmol Vis Sci 1995;36:906-13.

16. Cadera W, Vlirre E, Karcik S. Cine MRI of ocular motility. J Pediatr Ophthalmol Strabismus 1992;29: $120-2$. 